\title{
Inhibition of specific binding of okadaic acid to protein phosphatase 2A by microcystin-LR, calyculin-A and tautomycin: method of analysis of interactions of tight-binding ligands with target protein
}

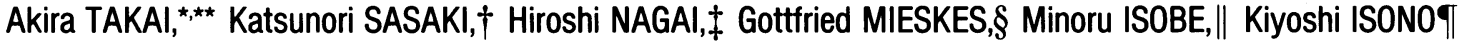 \\ and Takeshi YASUMOTO† \\ *Department of Physiology, School of Medicine, Nagoya University, 65 Tsurumai-cho, Showa-ku, Nagoya 466, Japan, \\ †Laboratory of Bioorganic Chemistry, Faculty of Agriculture, Tohoku University, 1-1 Tsutsumidori-Amemiyamachi, Aoba-ku, Sendai 981, Japan, \\ $\ddagger$ Department of Pathology, John A. Burns School of Medicine, University of Hawaii, 1960 East-West Road, Honolulu, Hawaii 96822, U.S.A., \\ §Abteilung Klinische Biochemie, Zentrum für Innere Medizin, Universität Göttingen, Robert-Koch-Straße 40, 37070 Göttingen, Germany, \\ |lLaboratory of Organic Chemistry, Faculty of Agriculture, Nagoya University, Furo-cho, Chikusa-ku, Nagoya 464-01, Japan, and \\ TDepartment of Marine Science, School of Marine Science and Technology, Tokai University, 3-20-1 Orido, Shimizu 424, Japan
}

Several groups have reported that okadaic acid (OA) and some other tight-binding protein phosphatase inhibitors including microcystin-LR (MCLR), calyculin-A and tautomycin prevent each other from binding to protein phosphatase 2A (PP2A). In this paper we have introduced an improved procedure for examining to what extent the affinity of an enzyme for a labelled tight-binding ligand is reduced by binding of an unlabelled tightbinding ligand to the enzyme. Using this procedure, we have analysed the dose-dependent reduction of PP2A binding of [24$\left.{ }^{3} \mathrm{H}\right] \mathrm{OA}$ by addition of OA, MCLR, calyculin-A and tautomycin. The results indicate that the binding of the unlabelled inhibitors to the PP2A molecule causes a dramatic $\left(10^{6}-10^{8}\right.$-fold $)$ increase in the dissociation constant associated with the interaction of $\left[24-{ }^{3} \mathrm{H}\right] \mathrm{OA}$ and PP2A. This suggests that OA and the other inhibitors bind to PP2A in a mutually exclusive manner. The protein phosphatase inhibitors may share the same binding site on the PP2A molecule. We have also measured values of the dissociation constant $\left(K_{\mathrm{i}}\right)$ for the interaction of these toxins with protein phosphatase 1 (PP1). For MCLR and calyculin-A, the ratio of the $K_{\mathrm{i}}$ value obtained for PP1 to that for PP2A was in the range 4-9, whereas it was $0.01-0.02$ for tuatomycin. The value of tautomycin is considerably smaller than that $(0.4)$ calculated from previously reported $K_{\mathrm{i}}$ values.

\section{INTRODUCTION}

Protein phosphatases 1 and 2A (PP1 and PP2A) are two of the four major enzymes that dephosphorylate serine/threonine residues of proteins in eukaryotic cells [see Cohen (1989) and Shenolikar and Nairn (1991)]. During the last decade these enzymes have been shown to be specifically inhibited by several naturally occurring toxins including okadaic acid (OA) (Takai et al., 1987; Bialojan and Takai, 1988), calyculin-A (Ishihara et al., 1989), microcystin-LR (MCLR) (MacKintosh et al., 1990), nodularin (Matsushima et al., 1990), tautomycin (MacKintosh and Klumpp, 1990) and cantharidin ( $\mathrm{Li}$ and Casida, 1992). These toxins are now used as powerful tools for research of an increasingly wide variety of cellular events regulated by reversible protein phosphorylation.

OA was the first to be identified as a substance of this category (Takai et al., 1987; Bialojan and Takai, 1988). The specificity and kinetics of phosphatase inhibition by this toxin have been studied most closely. In comparison with the other potent inhibitors subsequently described, OA is characterized by its much higher affinity for PP2A than for PP1. We have reported that $K_{\mathrm{i}}$ values for its interaction with PP1 and PP2A are of the order of $150 \mathrm{nM}$ and $30 \mathrm{pM}$ respectively (Takai and Mieskes, 1991; Takai et al., 1992a; see Table 3). The affinity of OA for PP2A is so high that it must be treated as a 'tight-binding inhibitor' [as defined by Henderson (1972)] when the steady-state dose-inhibition relationship is analysed (Takai and Mieskes, 1991; Takai et al., 1992a). Because experiments with tightbinding inhibitors often have to be performed with very low enzyme and inhibitor concentrations, the enzyme-inhibitor interaction tends to show a marked time-dependence (Cha, 1975). We have shown that the interaction of OA and PP2A is indeed very time-dependent (Takai et al., 1992b). On analysing the presteady-state time course of OA-PP2A interaction, we have found that the rate constants for binding of OA and PP2A are of the order of $10^{7} \mathrm{M}^{-1} \cdot \mathrm{s}^{-1}$, a typical value for reactions involving relatively large molecules, whereas those for their dissociation have very low values in the range $10^{-4}$ to $10^{-3} \mathrm{~s}^{-1}$.

Interestingly, experiments by several groups have shown that $\mathrm{OA}$ and the other inhibitors prevent each other from binding to PP2A. MacKintosh et al. (1990) have examined modification of the inhibitory effect of MCLR on PP1 by PP2A. They used a PP2A preparation that, although inactivated by pretreatment with $\mathrm{NaF}$ and pyrophosphate, still retained the ability to bind MCLR. Addition of the PP2A preparation caused an increase in the concentration of MCLR required for $50 \%$ inhibition of PP1. They have shown that this change in the affinity of MCLR is abolished by preincubating the PP2A preparation with OA. Using a similar methodology, they have also shown that OA prevents binding of tautomycin to PP2A (MacKintosh and Klumpp, 1990). Fujiki and Suganuma (1993) have reported that specific binding of an ${ }^{3} \mathrm{H}$-labelled $\mathrm{OA}$ preparation to a fraction

Abbreviations used: OA, okadaic acid; MCLR, microcystin-LR; PP1, protein phosphatase 1; PP2A, protein phosphatase 2A; pNPP, $p$-nitrophenyl phosphate; PMLC, phosphorylated myosin light chains; f.a.b.-ms, fast-atom-bombardment mass spectrometry.

** To whom correspondence should be addressed. 
of mouse brain containing protein phosphatases (mainly PP2A) is dose-dependently inhibited by MCLR, calyculin-A and tautomycin. Li and Casida (1992) have shown that binding of ${ }^{3} \mathrm{H}-$ labelled cantharidin to a mouse liver fraction containing PP2A is reduced in the presence of $O A$.

Such observations suggest that the protein phosphatase inhibitors may share the same binding site on the PP2A molecule. However, this attractive possibility has not been quantitatively examined so far. The major difficulty lies in the fact that OA and some of the other inhibitors act on PP2A as tight-binding inhibitors (Henderson, 1972) for which conventional methods of data analysis, based on Michaelis-Menten-type equations, are not applicable. For example, the standard procedure of the Scatchard (1949) plot cannot be applied for analysis of bindingassay data when two competitively binding ligands involved are tight-binding inhibitors (see Henderson, 1972).

In the present experiments we have introduced an improved method for examining to what extent the affinity of an enzyme for a labelled tight-binding ligand is reduced when the enzyme binds to an unlabelled tight-binding ligand. Using this method, we analysed the decrease in PP2A binding of $\left[24-{ }^{3} \mathrm{H}\right] \mathrm{OA}$ by addition of OA, MCLR, calyculin-A and tautomycin. To compare the ability of the unlabelled inhibitors to reduce the PP2A binding of $\left[24-{ }^{3} \mathrm{H}\right] \mathrm{OA}$ with their affinity for the enzyme, we measured the dissociation constants for the enzyme-inhibitor interaction by dose-inhibition analysis. As information on selectivity is useful for biochemical and physiological applications of inhibitors, dose-inhibition analysis was also carried out with PP1 as well as with PP2A. In previous work with OA and its derivatives, we have repeatedly noticed that the molar concentration of the enzyme is a crucially important parameter in experiments with tight-binding inhibitors (Takai and Mieskes, 1991; Takai et al., 1992a,b; Sasaki et al., 1994). In the present experiments therefore particular care was taken to make the enzyme concentration (and also the inhibitor concentrations) as accurate as possible. We have also considered the time-dependent nature of reactions involving tight-binding ligands.

We present here results that indicate that binding of the unlabelled inhibitors to PP2A causes a large $\left(10^{6}-10^{8}\right.$-fold $)$ increase in the dissociation constant associated with the interaction of $\left[24-{ }^{3} \mathrm{H}\right] \mathrm{OA}$ and PP2A. This suggests that PP2A binding of $\mathrm{OA}$ and that of the other inhibitors are mutually exclusive. The protein phosphatase inhibitors may indeed share the same binding site on the PP2A molecule.

\section{EXPERIMENTAL}

\section{Materials}

OA was kindly given by Dr. Y. Tsukitani (Fujisawa Pharmaceutical Co., Tokyo, Japan). MCLR and calyculin-A were purchased from Wako Pure Chemicals Co., Osaka, Japan. Tautomycin was purified from Streptomyces spiroverticillatus by the method described previously (Cheng et al., 1987, 1990). pNitrophenyl phosphate ( $p$ NPP; disodium salt) was a product of Sigma Chemical Co. (catalogue code, Sigma-104). $\left[\gamma^{-32}\right.$ P]ATP was purchased from Amersham Japan Co. All other chemical reagents used were of analytical grade.

The protein phosphatase inhibitors were dissolved in pure dimethyl sulphoxide and added to aqueous buffers. The maximal concentration of dimethyl sulphoxide in the reaction mixtures was $0.01 \%(\mathrm{v} / \mathrm{v})$. Control results were not affected by the addition of this amount of dimethyl sulphoxide. Concentrations of MCLR, calyculin-A and tautomycin were adjusted by a titration procedure using OA as standard (Takai et al., 1992a).

\section{Preparation and assay of protein phosphatases}

The catalytic subunits of PP2A and PP1 were prepared from rabbit skeletal muscle essentially as described by Tung et al. (1984). SDS/PAGE of the PP2A and PP1 preparations revealed single polypeptides of molecular mass $35-36 \mathrm{kDa}$. The molar concentration of the PP2A calculated from the protein concentration (Lowry et al., 1951) and the molecular mass [35.6 kDa (Cohen, 1989)] agreed well with that determined by the titration procedure using OA as standard (Takai and Mieskes, 1991). The PP1 preparation appeared to be somewhat less pure than the PP2A preparation, its molar concentration determined by the titration procedure with MCLR as standard being $60-70 \%$ of that calculated from the protein concentration. The activity of PP1 with phosphorylated myosin light chains (PMLC) (see below) was suppressed by $97 \pm 2 \%(n=8)$ in the presence of $100 \mathrm{nM}$ inhibitor 2 (Cohen et al., 1988). Myosin light chains were purified from chicken gizzard essentially as described for isolation of cardiac light chains by Cummins and Lambert (1986), and they were $\left[{ }^{32} \mathrm{P}\right]$ phosphorylated with chicken gizzard myosin lightchain kinase isolated by the method of Ngai et al. (1984). Phosphorylase $b$ and phosphorylase kinase from rabbit muscle, which were used for preparation of $\left[{ }^{32} \mathrm{P}\right]$ phosphorylated phosphorylase $a$ by the method of Antoniw et al. (1977), were purchased from Sigma.

The activities of the protein phosphatases were measured at $25^{\circ} \mathrm{C}$ as described previously (Bialojan and Takai, 1988; Takai et al., 1992a). The buffer solution used contained (mM): Tris (base), 40; $\mathrm{MgCl}_{2}, 34$; EDTA (free acid), 4, DL-dithiothreitol, 4. No bivalent cation other than $\mathrm{Mg}^{2+}$ was added. The solution was used without titration ( $\mathrm{pH} 8.4$ at $\left.25^{\circ} \mathrm{C}\right)$.

\section{Preparation of $\left[24-{ }^{3} \mathrm{H}\right] \mathrm{OA}$}

To synthesize $\left[24-{ }^{3} \mathrm{H}\right] \mathrm{OA}$ from OA we designed the following chemical protocol. OA was first converted into a mixture of the $p$-bromophenacyl ester forms of its dehydrogenated derivatives by the method described for preparation of 27-dehydro-OA (Sasaki et al., 1994). p-Bromophenacyl-24-dehydro-OA was then separated from the mixture by h.p.l.c. on a reversedphase column (Cosmosil ODS5 C18-AR; internal diameter $10 \mathrm{~mm} \times 250 \mathrm{~mm}$; Nacalai Tesque, Kyoto, Japan) with acetonitrile/water $(9: 1, \mathrm{v} / \mathrm{v})$ as the mobile phase at a flow rate of $2 \mathrm{ml} / \mathrm{min}$. The 24-dehydro-OA ester was eluted at $27.8 \mathrm{ml}$ (yield, $33.4 \%$ ) and the 27-dehydro-OA ester at $32.4 \mathrm{ml}$ (yield, $12.1 \%$ ). Fast-atom-bombardment mass spectroscopy (f.a.b.-m.s.) of the 24-dehydro-OA ester revealed ions at $m / z 999$ and 1001, corresponding to the protonated molecular species containing the natural bromine isotopes ${ }^{79} \mathrm{Br}$ and ${ }^{81} \mathrm{Br}$ respectively. In the ${ }^{1} \mathrm{H}$ n.m.r. spectrum (400 MHz, in [ $\left.{ }^{2} \mathrm{H}\right]$ acetonitrile) the signal due to the 24-H of OA (which is adjacent to the 24-OH) disappeared after the oxidation procedure. The triplet signal ( $\delta 3.24$ p.p.m.) due to the $23-\mathrm{H}$ of $p$-bromophenacyl-OA was changed to a broad singlet and shifted downfield ( $\delta 4.17$ p.p.m.). These spectral data are consistent with the structural modification of the 24 position.

The 24-dehydro-OA ester $(1.1 \mu \mathrm{mol})$ was then reduced with $\mathrm{NaBH}_{4}(21.2 \mu \mathrm{mol})$ at $-15^{\circ} \mathrm{C}$ for $1 \mathrm{~h}$ in $500 \mu \mathrm{l}$ of methanol containing $21.2 \mu \mathrm{mol}$ of $\mathrm{CeCl}_{3}$. The reaction was stopped with $100 \mu \mathrm{l}$ of acetone and the solution was kept at room temperature for $30 \mathrm{~min}$. It was then diluted with distilled water and mixed with ethyl acetate to extract reaction products in the organic phase. The sample obtained was subjected to reversed-phase h.p.l.c. using the same column conditions as described above to give the major component (yield, $70 \%$ ) which was eluted at $21.2 \mathrm{ml}$. F.a.b.-m.s. of the product showed two sets of ions corresponding to protonated and cationated molecular species at 
$m / z 1003$ and 1005 and $m / z 1025$ and 1027 respectively, indicating that the carbonyl groups in both the $p$-bromophenacyl and OA moieties of the ester were reduced to hydroxyls. This structure was confirmed by the ${ }^{1} \mathrm{H}$-n.m.r. spectrum, which exhibited signals related to $2^{\prime}-\mathrm{H}$ (m, 4.87 p.p.m.), 24-H (d, 4.05 p.p.m.) and 24$\mathrm{OH}\left(\delta 3.58\right.$ p.p.m.). The split signals of the $2^{\prime}-\mathrm{OH}(\delta 3.90$ p.p.m. and 4.00 p.p.m.) indicated that the reduction product was a mixture of stereoisomers at the $2^{\prime}-\mathrm{C}$.

The ester bond of the reduced product $(0.29 \mu \mathrm{mol})$ was hydrolysed with $0.2 \mathrm{M} \mathrm{NaOH}$ in methanol/water $(8: 2, \mathrm{v} / \mathrm{v})$ at $60{ }^{\circ} \mathrm{C}$ for $2 \mathrm{~h}$. The solution was then diluted with distilled water and the $\mathrm{pH}$ was adjusted to 6.0 with acetic acid. After extraction with ethyl acetate, the sample was again applied to h.p.l.c. on the reversed-phase column (see above) with acetonitrile/water $(7: 3$, $\mathrm{v} / \mathrm{v}$ ) containing $0.05 \%$ acetic acid as mobile phase. OA $(0.26 \mu \mathrm{mol}=90 \%)$ was eluted at $25 \mathrm{ml}$. F.a.b.-m.s. of the recovered OA revealed a deprotonated molecular ion at $m / z 803$, as observed with the original sample of OA. In the ${ }^{1}$ H-n.m.r. spectrum of the recovered sample, the signal due to the $23-\mathrm{H}$ appeared as a triplet $(J 12 \mathrm{~Hz})$ at $\delta 3.26$ p.p.m., indicating that the equatorial configuration of the $24-\mathrm{OH}$ was preserved. [If the 24-OH were in an axial position, the $23-\mathrm{H}$ should have given a quartet $(J 12,4 \mathrm{~Hz})$.] These spectral data confirm that the series of reactions, as a whole, did not alter the chemical structure of OA.

The above scheme, which we developed using unlabelled reactants, was applied to prepare $\left[24-{ }^{3} \mathrm{H}\right] \mathrm{OA}(130 \mathrm{GBq} / \mathrm{mmol}$; see the Results section for the procedure of the determination of this value) from $p$-bromophenacyl-24-dehydro-OA (at DuPontNEN) using $\mathrm{NaB}^{3} \mathrm{H}_{4}$ as the reductant in place of $\mathrm{NaBH}_{4}$. The molar concentration of $\left[24-{ }^{3} \mathrm{H}\right] \mathrm{OA}$ was adjusted by measuring its binding affinity to PP2A using unlabelled OA as standard (see the Results section).

\section{Binding assay}

The binding assay was carried out at $25^{\circ} \mathrm{C}$ with the same buffer as that used for the assay of the phosphatase activities (see above). $\left[24-{ }^{3} \mathrm{H}\right] \mathrm{OA}$ and unlabelled phosphatase inhibitors were first added to the buffer in reaction tubes. To start the reaction, PP2A ( $2.0 \mathrm{nM}$; final concentration) was added to each tube and the contents mixed vigorously. [We chose this order of addition because OA (probably also $\left[24-{ }^{3} \mathrm{H}\right] \mathrm{OA}$ ) dissociates from PP2A very slowly once it is bound to the enzyme. We have reported that the rate constants associated with dissociation of $\mathrm{OA}$ from PP2A are of the order of $10^{-3}-10^{-4} \mathrm{~s}^{-1}$ (Takai et al., 1992b).] After incubation for $30 \mathrm{~min}$, each sample $(1.0 \mathrm{ml})$ was decanted into one of the ten inlets of a VF-10N vacuum filter holder (Sanplatec Co., Osaka, Japan) equipped with a Whatman GF/C glass filter (diameter $24 \mathrm{~mm}$ ). The filters were washed by adding $1 \mathrm{ml}$ of the buffer from the inlets five times. [ $p$ NPP phosphatase activity in the filtrate was undetectably low, confirming that binding of PP2A to the filter was sufficiently stable.] After drying, the filters were placed in scintillation vials with $4 \mathrm{ml}$ of Econofluor-2 scintillation cocktail (DuPont-NEN) for determination of radioactivity bound. We have shown that binding of nanomolar concentrations of OA and PP2A is highly timedependent [see Figure 1 of Takai et al. (1992b)]. From the spectrophotometrically observed time course of inhibition of the $p$ NPP phosphatase activity of PP2A by the unlabelled inhibitors, the above reaction time $(30 \mathrm{~min})$ should be long enough for a state of equilibrium to be established between the enzyme and inhibitors.

In preliminary experiments we noticed that there were two distinct types of non-specific binding of radioactivity to the filter.
(1) A small amount of radioactivity remained on the filter even when a high concentration $(20 \mu \mathrm{M})$ of unlabelled OA was added to the reaction mixture. Although the amount of the remaining radioactivity was linearly correlated with the concentration of $\left[24-{ }^{3} \mathrm{H}\right] \mathrm{OA}$ added, it was not significantly affected by changing the concentration of PP2A (0-10 nM) and/or that of unlabelled OA $(5-100 \mu \mathrm{M})$. This remaining activity, which we simply subtracted from the original data, seems to be due to a small fraction of ${ }^{3} \mathrm{H}$ that was not incorporated into OA. (2) A second type of residual radioactivity remained on the filter even when no enzyme was present in the reaction mixture. This radioactivity was not easily decreased by additional washing of the filter, whereas it was completely eliminated by adding excess $(10-50 \mu \mathrm{M})$ unlabelled $\mathrm{OA}$ to the reaction mixture. These observations indicate that $\left[24-{ }^{3} \mathrm{H}\right] \mathrm{OA}$ has some affinity for the filter. There was a linear correlation between the concentration of $\left[24-{ }^{3} \mathrm{H}\right] \mathrm{OA}$ and the amount of residual binding when the concentration of $\left[24-{ }^{3} \mathrm{H}\right] \mathrm{OA}$ was in the range $0-10 \mathrm{nM}$. This type of 'non-specific' binding was subtracted from the data by the method described below (see the Theory section). Unfortunately, the residual binding increased steeply and became very variable when the concentration of $\left[24-{ }^{3} \mathrm{H}\right] \mathrm{OA}$ exceeded $20 \mathrm{nM}$. For this reason we could not use this binding-assay method for PP1, which exhibits a nearly 5000-fold weaker affinity for OA than does PP2A (see Table 3).

\section{THEORY}

The symbols used are defined in Table 1.

\section{Dose-inhibition analysis}

To describe steady-state dose-inhibition relationships for tightbinding inhibitors we use the model function:

$\zeta\left(I_{\mathrm{t}}\right)=\frac{E_{\mathrm{t}}-I_{\mathrm{t}}-K_{\mathrm{i}}+\sqrt{ }\left[\left(E_{\mathrm{t}}-I_{\mathrm{t}}-K_{\mathrm{i}}\right)^{2}+4 E_{\mathrm{t}} K_{\mathrm{i}}\right]}{2 E_{\mathrm{t}}}$

where $\zeta\left(I_{t}\right)$ denotes the fractional activity (i.e. the ratio of the enzyme activity in the presence of the inhibitor to that in its absence) at a given total inhibitor concentration, $I_{t}$. This function is readily derived using steady-state assumptions, considering the reduction of the free inhibitor concentration to be caused by

\section{Table 1 Symbols used and their definitions}

\begin{tabular}{ll}
\hline Symbol & Definition \\
\hline$E$ & Enzyme \\
$E_{\mathrm{t}}$ & Total concentration of enzyme \\
$I_{\mathrm{t}}$ & Total concentration of inhibitor \\
$\mathrm{L}_{0}, L_{0}$ & Unlabelled ligand and its total concentration \\
$\mathrm{L}_{1}, L_{1}$ & Isotope-labelled ligand and its total concentration \\
$K_{\mathrm{i}}$ & Dissociation constant for enzyme-inhibitor interaction \\
$K_{0}$ & Dissociation constant for $E-L_{0}$ interaction \\
$K_{1}$ & Dissociation constant for $E-L_{1}$ interaction \\
$K_{0}^{\prime}$ & Dissociation constant for $E L_{1}-L_{0}$ interaction \\
$K_{1}^{\prime}$ & Dissociation constant for $E L_{0}-L_{1}$ interaction \\
$K$ & $=K_{0}^{\prime} / K_{0}=K_{1}^{\prime} / K_{1}$ \\
$\zeta\left(I_{\mathrm{t}}\right)$ & Fractional activity of $E$ at a given total inhibitor concentration, $I_{\mathrm{t}}$ \\
$\phi$ & Concentration of $L_{1}-$ bound form of $E$ \\
$\bar{\nu}$ & Conventional notation for $\phi / E_{1}$, used in the Scatchard analysis \\
$N$ & Specific radioactivity of $L_{1}$ \\
$\epsilon$ & Radioactivity associated with non-specific binding of $L_{1}$ to filter \\
$\Phi$ & Radioactivity of enzyme-bound $L_{1}$ plus $\epsilon$
\end{tabular}


binding with the enzyme (Straus and Goldstein, 1943; Cha, 1970; Henderson, 1972). We have shown that eqn. (1) fits well the steady-state dose-inhibition relationships for the interaction of PP2A with OA and its derivatives (Takai and Mieskes, 1991; Takai et al., 1992a,b).

\section{Binding assay}

Binding of an isotope-labelled ligand in the absence of other ligands

When an isotope-labelled ligand, $\mathrm{L}_{1}$, interacts with $\mathrm{E}$ in the absence of unlabelled ligand, the steady-state concentration of the $L_{1}$-bound form of $\mathrm{E}, \phi$, is given as an explicit function of $L_{1}$, which is closely related to eqn. (1):

$\phi=\left[\mathrm{EL}_{1}\right]=\frac{E_{\mathrm{t}}+L_{1}+K_{1}-\sqrt{ }\left[\left(E_{\mathrm{t}}+L_{1}+K_{1}\right)^{2}-4 E_{\mathrm{t}} L_{1}\right]}{2}$

Note that, in this case,

$E_{\mathrm{t}}=[\mathrm{E}]+\left[\mathrm{EL}_{1}\right]$

Rearranging eqn. (2), we have

$\frac{\bar{\nu}}{\left[\mathrm{L}_{1}\right]}=\frac{1}{K_{1}}(1-\bar{v})$

where $\bar{\nu}=\left[\mathrm{EL}_{1}\right] / E_{\mathrm{t}}$ and $\left[\mathrm{L}_{1}\right]=L_{1}-\left[\mathrm{EL}_{1}\right]$. Therefore the plot of $\bar{\nu} /\left[\mathrm{L}_{1}\right]$ against $\bar{\nu}$ (i.e. Scatchard plot) becomes linear in this case whether or not $\mathrm{L}_{1}$ is a tight-binding inhibitor.

Mathematically, eqn. (4) is equivalent to eqn. (2). However, the left-hand side of eqn. (4), $\bar{\nu} /\left[\mathrm{L}_{1}\right]$, frequently becomes very vulnerable because $\left[\mathrm{L}_{1}\right]$ tends to be close to zero when $L_{1}$ is a tight-binding inhibitor. Furthermore, transformation of data like eqn. (4) often leads to a serious bias [see e.g. pp. 180-217 of Sen and Srivastava (1990)]. As pointed out by Henderson (1972), it is generally very difficult to calculate the correct weighting factors for terms of transformed equations used in linear replotting procedures because of the stochastic dependence of the variables. For these reasons, we use explicit forms of model functions [e.g. eqn. (2)] rather than their transformations [e.g. eqn. (4)] for data analysis throughout the present paper.

\section{General interaction model}

Interaction of an enzyme, $E$, with a labelled ligand, $L_{1}$, in the presence of an unlabelled ligand, $\mathrm{L}_{0}$, is represented by a general interaction model as shown in Scheme 1. When both $\mathrm{L}_{0}$ and $\mathrm{L}_{1}$ are tight-binding ligands, the explicit form of $\phi$ as a function of $E_{\mathrm{t}}, L_{0}$ and $L_{1}$ is derived, as follows. By definition we have:

$\phi=\left[\mathrm{EL}_{1}\right]+\left[\mathrm{EL}_{0} \mathrm{~L}_{1}\right]$

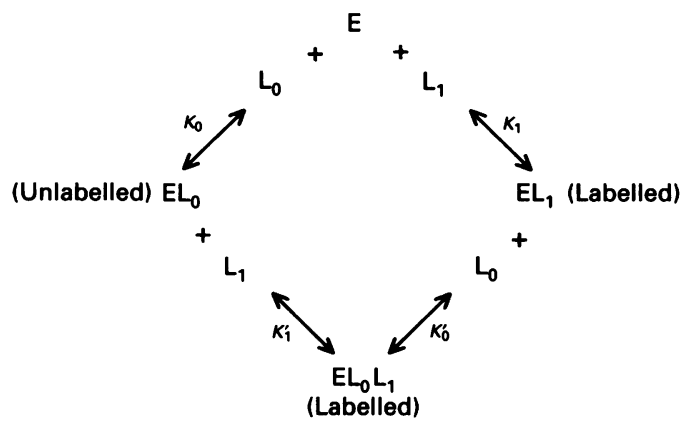

The conservation equations for $\mathrm{E}, \mathrm{L}_{0}$ and $\mathrm{L}_{1}$ are

$E_{\mathrm{t}}=[\mathrm{E}]+\left[\mathrm{EL}_{0}\right]+\left[\mathrm{EL}_{1}\right]+\left[\mathrm{EL}_{0} \mathrm{~L}_{1}\right]$

$L_{0}=\left[\mathrm{L}_{0}\right]+\left[\mathrm{EL}_{0}\right]+\left[\mathrm{EL}_{0} \mathrm{~L}_{1}\right]$

and

$L_{1}=\left[\mathrm{L}_{1}\right]+\left[\mathrm{EL}_{1}\right]+\left[\mathrm{EL}_{0} \mathrm{~L}_{1}\right]$

In reactions of ligands with relatively low affinity, some or all of the three terms, $\left[E L_{0}\right],\left[E L_{1}\right]$ and $\left[E L_{0} L_{1}\right]$, in eqn. (7) and/or eqn. (8) can be assumed to be negligible. (For example, the term corresponding to $\left[E L_{0} L_{1}\right]$ in eqn. (8) [but not that in eqn. (7)] is neglected in the derivation of eqns. (A3)-(A6) of Henderson (1972).\} However, this Michaelis-Menten-type assumption is often seriously violated with reactions involving tight-binding ligands.

Assuming a state of equilibrium we have:

$K_{0}=\frac{[\mathrm{E}]\left[\mathrm{L}_{0}\right]}{\left[\mathrm{EL}_{0}\right]}$

$K_{1}=\frac{[\mathrm{E}]\left[\mathrm{L}_{1}\right]}{\left[\mathrm{EL}_{1}\right]}$

and

$K_{0}^{\prime}=k K_{0}=\frac{\left[\mathrm{EL}_{1}\right]\left[\mathrm{L}_{0}\right]}{\left[\mathrm{EL}_{0} \mathrm{~L}_{1}\right]}$

Note that

$k=\frac{K_{0}^{\prime}}{K_{0}}=\frac{K_{1}^{\prime}}{K_{1}}$

We can give a thermodynamic interpretation to $k$ as follows. When one of the two ligands binds to the enzyme, the absolute value of the standard free-energy change for the interaction of the enzyme with the other ligand decreases by:

$\delta \Delta G^{0 \prime}=R T \ln k$

Elimination of $\left[\mathrm{L}_{0}\right],\left[\mathrm{L}_{1}\right],\left[E \mathrm{~L}_{0}\right]$ and $\left[E L_{1}\right]$ from eqns. (5)-(12) gives a quintic equation in $\phi$ (not shown), for which no algebraic solution exists. [This is the basic reason why the steady-state kinetics of this type of model system cannot be simply analysed by ordinary linear-replotting procedures (e.g. Scatchard plot).] But if we replace eqn. (6) with:

$E_{\mathrm{t}}=\left[\mathrm{EL}_{0}\right]+\left[\mathrm{EL}_{1}\right]+\left[\mathrm{EL}_{0} \mathrm{~L}_{1}\right]$,

the equation is reduced to a quartic of the following form, which can be solved explicitly using the familiar formula (see below):

$\phi^{4}+a \phi^{3}+b \phi^{2}+c \phi+d=0$

where

$a=-2\left(E_{\mathrm{t}}+K_{1} k+L_{1}\right)$

$b=K_{1}\left(K_{1}-K_{0}\right) k^{2}+K_{1}\left(3 E_{\mathrm{t}}-L_{0}+2 L_{1}\right) k+E_{\mathrm{t}}^{2}+4 E_{\mathrm{t}} L_{1}+L_{1}^{2}$

$c=K_{1}\left[E_{t}\left(K_{0}-K_{1}\right)+K_{1} L_{0}+K_{0} L_{1}\right] k^{2}$ $-K_{1}\left(E_{\mathrm{t}}^{2}-E_{\mathrm{t}} L_{0}+3 E_{\mathrm{t}} L_{1}-L_{0} L_{1}\right) k-2 E_{\mathrm{t}} L_{1}\left(E_{\mathrm{t}}+L_{1}\right)$

and

$d=-E_{\mathrm{t}} L_{1}\left[K_{0} K_{1} k^{2}+K_{1}\left(L_{0}-E_{\mathrm{t}}\right) k-E_{\mathrm{t}} L_{1}\right]$

If $L_{1}$ is a tight-binding ligand of $E$, it is not difficult to find an experimental condition under which we may neglect the [E] term in eqn. (6) and use eqn. (14) in its place. For example, let $E$ and $\mathrm{L}_{1}$ be PP2A and $\left[24-{ }^{3} \mathrm{H}\right] \mathrm{OA}$ at concentrations of (say) $2 \mathrm{nM}$ and $5 \mathrm{nM}$ respectively. Then, because $K_{1}=32 \mathrm{pM}$ in this case (see Table 3), it follows from eqns. (2) and (3) that $[\mathrm{E}] \cong 0.01 E_{\mathrm{t}}$ in the absence of $\mathrm{L}_{0}$. When $k \geqslant 1$, it can be shown mathematically that 
[E] is unchanged or decreased (but never increased) from this level by addition of any concentration of $\mathrm{L}_{0}$.

To solve eqn. (15) by the formula for roots of quartic equations [see e.g. pp. 102-103 and 121-122 of Birkhoff and MacLane (1977)], we define the following parameters:

$$
\begin{aligned}
& p=-\frac{3 a^{2}}{8}+b, q=\frac{a^{3}}{8}-\frac{a b}{2}+c, r=-\frac{3 a^{4}}{256}+\frac{a^{2} b}{16}-\frac{a c}{4}+d, \\
& s=-\frac{p^{2}}{3}-4 r, t=\frac{2 p^{3}}{27}+q^{2}-\frac{8 p r}{3}, u=\frac{t / 2}{|s / 3|^{3 / 2}} \\
& v=\frac{p}{3}+\cos \left(\frac{\arccos u}{3}\right), A=\sqrt{ }(v-p) \text { and } B=\frac{q}{2 A}
\end{aligned}
$$

Then, noting that $0 \leqslant \phi<E_{\mathrm{t}}$, we have:

$$
\phi=\phi\left(E_{\mathrm{t}}, L_{0}, L_{1}\right)=-\frac{a}{4}+\frac{-A+\sqrt{ }\left[A^{2}+4\left(B-\frac{v}{2}\right)\right]}{2}
$$

In the present binding assays we measure the dependence of $\phi$ on $L_{0}$ at fixed $E_{\mathrm{t}}$ and $L_{1}$. [The values of $E_{\mathrm{t}}$ and $L_{1}$ are set so that eqn. (14) can be used in place of eqn. (6) (see above).]. We then fit eqn. (16) to the sets of $\left(L_{0}, \phi\right)$ data obtained and thereby estimate $k$ and $K_{0}$. Ordinary computer programs for leastsquares fitting do not accept a model function which requires computation of complex numbers in intermediary steps. Fortunately it can be proved that all the above parameters including $A$ are real for any (positive) values of $E_{\mathrm{t}}, L_{0}, L_{1}, K_{0}, K_{1}$ and $k$.

\section{Competitive binding model}

Competitive interaction of two ligands (Scheme 2) can be defined as a limiting case of the general interaction model, where $k=\infty$ and therefore $\left[\mathrm{EL}_{0} \mathrm{~L}_{1}\right]=0$.

In this case eqn. (15) reduces to a quadratic:

$$
b^{\prime} \phi^{2}+c^{\prime} \phi+d^{\prime}=0
$$

where

$$
\begin{aligned}
b^{\prime} & =K_{1}-K_{0} \\
c^{\prime} & =E_{\mathrm{t}}\left(K_{0}-K_{1}\right)+K_{1} L_{0}+K_{0} L_{1} \\
d^{\prime} & =-E_{\mathrm{t}} L_{1} K_{0} \\
& \text { If } K_{0}=K_{1} \text {, the solution of eqn. (17) is: }
\end{aligned}
$$

$$
\phi=\frac{L_{1}}{L_{0}+L_{1}} E_{\mathrm{t}}
$$

In the present paper we use eqn. (18) to describe the PP2A binding of $\left[24-{ }^{3} \mathrm{H}\right] \mathrm{OA}$ in the presence of unlabelled OA.

If $K_{0} \neq K_{1}$, then solving eqn. (17) and recalling that $0 \leqslant \phi<E_{\mathrm{t}}$, we have:

$$
\phi=\frac{-c^{\prime}+\sqrt{ }\left(c^{2}-4 b^{\prime} d^{\prime}\right)}{2 b^{\prime}}
$$

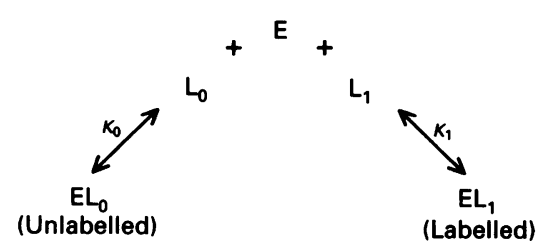

We use eqn. (19) as an explicit form of $\phi$ for regression analysis of interaction of $\left[24-{ }^{3} \mathrm{H}\right] \mathrm{OA}$ with MCLR, calyculin-A or tautomycin on PP2A.

Li et al. (1993) have successfully used the standard procedure of Scatchard analysis to examine the competitive interaction of $\left[{ }^{3} \mathrm{H}\right]$ cantharidin and some low-affinity ligands with PP2A. However, such a procedure is not applicable for analysis of the present binding-assay data, because it is shown that the $\left(\bar{\nu}, \bar{\nu} /\left[L_{1}\right]\right)$ relationship is not linear when $\mathrm{L}_{0}$ is a tight-binding ligand [see Figure 9 of Henderson (1972)].

\section{Non-specific binding of isotope-labelled ligand}

The radioactivity, $\Phi$, measured by the present filter method (see the Experimental section) is given by:

$\Phi=N w \phi+\epsilon$

where $N$ is the specific radioactivity (per mol of $\mathrm{L}_{1}$ ), $w$ is the volume of the reaction mixture, and $\epsilon$ is the non-specific binding of $\mathrm{L}_{1}$ to the filter. Assuming that $\epsilon$ is proportional to $\left[\mathrm{L}_{1}\right]$ (rather than to the total concentration $L_{1}$ ), we have

$\epsilon=\gamma N w\left(L_{1}-\phi\right)$

where $\gamma$ is the constant of proportionality. Note that $\epsilon$ is dependent on $L_{0}$ as well as on $L_{1}$.

We have estimated the value of $\gamma$ for $\left[24-{ }^{3} \mathrm{H}\right] \mathrm{OA}(0-10 \mathrm{nM})$ at $0.01-0.02$ by measuring the binding of radioactivity to filters in the absence of the enzyme where $\phi=0$ and $L_{1}=\left[\mathrm{L}_{1}\right]$ (see the Experimental section).

\section{Fitting and statistics}

The model functions were fitted to data by non-linear leastsquares regression with the use of the Origin software package, version 3.5 (MicroCal Software, Northampton, MA, U.S.A.), which utilizes the Levenberg-Marquardt algorithm [see e.g. p. 302 of Sen and Srivastava (1990)]. In the fitting we used as weight the reciprocal of the square of the standard error at each value of the independent variable [see pp. 9-15 of Carroll and Ruppert (1988)]. In previous dose-inhibition studies (Takai et al., 1992a,b; Sasaki et al., 1994) we used a non-parametric regression procedure for fitting eqn. (1) to the data. The previous and present fitting methods gave very similar estimations of $K_{1}$ as long as the number of experiments was sufficiently large. The values of $K_{1}$ and $K_{0}$ with S.E.M. obtained by the non-linear least-squares fitting were compared by a modified $t$ test [see pp. 15-18 of Carroll and Ruppert (1988)], which is based on a large-sample assumption of Wald (1943). Equality of variance was tested by an ordinary method using the $F$ statistic [see e.g. pp. 98-102 of Snedecor and Cochran (1980)]. In both cases, differences were taken as statistically significant when a two-tailed probability of less than 0.05 was obtained.

\section{RESULTS}

\section{Dose-inhibition analysis}

Table 2 gives the specific activities of the phosphatase preparations against $p \mathrm{NPP}(5 \mathrm{mM}), \operatorname{PMLC}(4 \mu \mathrm{M})$ and phosphorylase $a(4 \mu \mathrm{M})$ which were measured at $25^{\circ} \mathrm{C}$ in the absence of inhibitors. The $\mathrm{pH}$ of the reaction mixture was 8.4 (see the Experimental section). All the present dose-inhibition experiments and binding assays were carried out at this $\mathrm{pH}$ which is optimal for the $p$ NPP phosphatase activities (Takai and Mieskes, 1991). The activities of the enzymes towards the phosphorylated 


\section{Table 2 Activities of PP1 and PP2A}

The activities of the PP2A and PP1 preparation were measured at $25^{\circ} \mathrm{C}$ and pH 8.4 with $p$ NPP $(5 \mathrm{mM})$, PMLC $(4 \mu \mathrm{M})$ and phosphorylase $a(4 \mu \mathrm{M})$ as substrates in the absence of inhibitors. The unit of activity is $\mu \mathrm{mol}$ of $\mathrm{P}_{\mathrm{i}} / \mathrm{min}$ per $\mathrm{mg}$ of protein. Results are means \pm S.E.M. and $n$ denotes the total number of experiments.

\begin{tabular}{llllll}
\hline & \multicolumn{2}{l}{ PP1 } & & \multicolumn{2}{l}{ PP2 } \\
\cline { 2 - 3 } \cline { 5 - 6 } Substrate & Activity & $n$ & & Activity & $n$ \\
\hline$p N P P$ & $0.14 \pm 0.01$ & 206 & & $48.6 \pm 0.5$ & 441 \\
PMLC & $0.95 \pm 0.13$ & 18 & & $7.1 \pm 1.2$ & 40 \\
Phosphorylase $a$ & - & - & & $1.4 \pm 0.2$ & 32 \\
\end{tabular}

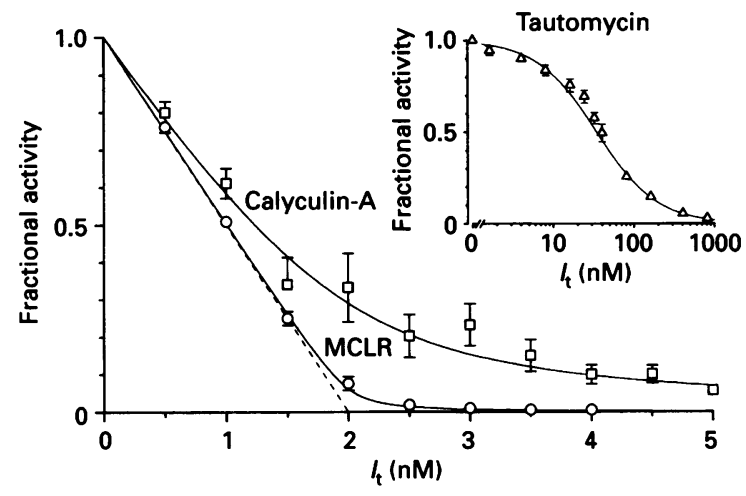

Figure 1 Inhibition of PP2A by MCLR, calyculin-A and tautomycin

Inhibitory effects of MCLR $(O)$, calyculin-A $(\square)$ and tautomycin $(\triangle)$ on the steady-state activity of PP2A with $p N P P(4 \mathrm{mM})$ as substrate were examined. Experiments were performed with a constant molar concentration $(2.0 \mathrm{nM})$ of PP2A. The intercept of the dashed line on the abscissa corresponds to $E_{1}$. Activities are presented as ratios to the values obtained in the absence of inhibitors. The data for tautomycin (inset) are plotted against the total inhibitor concentration $l_{t}$ on a logarithmic scale. For MCLR and calyculin-A, the scale of $l_{t}$ is linear. The curves are least-squares fits of eqn. (1) to the data. Results are means of data with S.E.M. if larger than symbol size. The number of experiments is listed in Table 3, which also gives the $K_{\mathrm{i}}$ values estimated by the fitting procedure.

proteins were about twice as high as those listed in Table 2 when the $\mathrm{pH}$ was lowered to 7.0. However, the phosphatases are almost inactive with $p$ NPP at this pH (Takai and Mieskes, 1991).

Figure 1 shows the relationship between the concentration of the phosphatase inhibitors applied and the steady-state activity of PP2A and $p$ NPP $(5 \mathrm{mM})$ as substrate. In these experiments the total molar concentration of PP2A in the reaction mixtures was kept constant at $2.0 \mathrm{nM}$ on the basis of the $E_{\mathrm{t}}$ value determined by fitting eqn. (1) to the dose-inhibition data for OA [data not shown; for typical dose-response curves for inhibition of PP2A by OA, see Takai and Mieskes (1991) and Takai et al. (1992a)]. The dose-inhibition data appear to be well described by eqn. (1) with the use of the $E_{\mathrm{t}}$ value (Figure 1). The values of $K_{\mathrm{i}}$ obtained by the fitting are listed in Table 3.

We also examined the inhibitory action of MCLR, calyculinA and tautomycin on the activity of PP2A $(2.0 \mathrm{nM})$ towards PMLC $(4 \mu \mathrm{M})$ and phosphorylase $a(4 \mu \mathrm{M})$ as well as on the $p$ NPP phosphatase activity. The $K_{\mathrm{i}}$ values obtained thereby are listed in Table 3, which shows that the susceptibility of the $p$ NPP phosphatase activity of PP2A to these inhibitors was not significantly different from that of its activities towards PMLC and phosphorylase $a$. In previous papers we have obtained very similar $K_{\mathrm{i}}$ values for $p$ NPP phosphatase activity of PP2A and for its phosphoprotein phosphatase activity (Takai and Mieskes, 1991; Takai et al., 1992a; Sasaki et al., 1994). Thus $p$ NPP seems to be an adequate substrate to use to compare the PP2A affinity of the protein phosphatase inhibitors. Dephosphorylation of $p$ NPP by PP2A, which has very high activity with this artificial substrate, can be determined easily and accurately by measuring the rate of production of $p$-nitrophenol with an ordinary spectrophotometer [Takai and Mieskes (1991); for the $p$ NPP phosphatase activity of PP2A, see also Goris et al. (1989) and Cayla et al. (1990)]. This is an advantageous feature of $p$ NPP over $\left[{ }^{32} \mathrm{P}\right]$ phosphorylated proteins as a substrate of PP2A for quantitative analysis.

Of the protein phosphatase inhibitors examined, MCLR exhibited the highest affinity for PP2A (Table 3). The value of $K_{1}$ for the interaction of this inhibitor with PP2A was of the order of $10 \mathrm{pM}$ (Table 3). Because of the analytical nature of eqn. (1), estimation of $K_{\mathrm{i}}$ from dose-inhibition data is not easy when $E_{\mathrm{t}}$ is much greater than $K_{\mathrm{r}}$. Generally speaking, $E_{\mathrm{t}} / K_{\mathrm{i}}$ should be as small as possible for the purpose of estimating $K_{\mathrm{i}}$ [see eqn. (6) of Takai and Mieskes (1991)]. Therefore we also examined the inhibitory effect of MCLR on the activity of PP2A to the phosphorylated proteins at a lower enzyme concentration $\left(E_{\mathrm{t}}=0.5 \mathrm{nM}\right)$. The $K_{\mathrm{i}}$ values obtained with $0.5 \mathrm{nM}$ PP2A using PMLC $(4 \mu \mathrm{M})$ and phosphorylase $a(4 \mu \mathrm{M})$ as substrates were $11 \pm 4 \mathrm{pM}(n=48)$ and $10 \pm 3 \mathrm{pM}(n=48)$ respectively. These values are in close agreement with those obtained with $2 \mathrm{nM}$ PP2A (Table 3).

Table 3 includes the $K_{\mathrm{i}}$ values obtained by dose-inhibition analysis with PP1 (1.8-2.5 nM) using $p$ NPP ( $5 \mathrm{mM})$ and PMLC $(4 \mu \mathrm{M})$ as substrates. Again we found no significant difference between the $K_{\mathrm{i}}$ values obtained with the different types of substrate. Of the inhibitors examined, it was also MCLR that exhibited the highest affinity for PP1.

$K_{1}$ values for interaction of OA with PP1 and PP2A (Table 3) agreed well with those reported in our previous papers (Takai and Mieskes, 1991; Takai et al., 1992a; Sasaki et al., 1994). The ratio of the $K_{\mathrm{i}}$ value for PP1 to that for PP2A (PP1/PP2A) is calculated at 4600 (Table 3), reconfirming that OA exhibits a much higher affinity for PP2A then it does for PP1 [see e.g. Takai et al. (1992a)]. Although MCLR exhibited very strong inhibitory activities towards both PP1 and PP2A, its affinity for PP1 was significantly weaker than for PP2A. Calyculin-A also exhibited a significantly higher affinity for PP2A than for PP1. However, the PP1/PP2A ratios for these two inhibitors, which fell in the range 4-9, were much smaller than the value for OA. In contrast with the other three inhibitors examined, tautomycin showed higher affinity for PP1 than for PP2A (Table 3). The $K_{\mathrm{i}}$ values (0.4-0.5 nM) obtained in the present experiments for this inhibitor with PP1 are comparable with that $(0.16 \mathrm{nM})$ reported by MacKintosh and Klumpp (1990). With PP2A, however, we obtained considerably larger $K_{\mathrm{i}}$ values $(26-35 \mathrm{nM})$ than they did $(0.4 \mathrm{nM})$. As a consequence, the value $(0.01-0.02)$ of the PP1/PP2A ratio for tautomycin listed in Table 3 is notably smaller than that $(0.4)$ calculated with the $K_{\mathrm{i}}$ values of MacKintosh and Klumpp (1990).

For the inhibition of PP2A by calyculin-A (Figure 1) the variation in data appeared to be larger at medium inhibitor concentrations $(1.5-3.5 \mathrm{nM})$ than the dose-inhibition relationships for the other inhibitors. A similar tendency was observed for the phosphoprotein phosphatase activities as well as for the $p$ NPP phosphatase activity of PP2A (results not shown). The larger variability of the dose-inhibition relationship observed with PP2A is possibly due to hydrolytic degradation of calyculinA, a monophosphate ester, by the remaining PP2A activity (see 


\section{Table 3 Dissociation constants for the interaction of PP2A with the protein phosphatase inhibitors}

The values for dissociation constants (means \pm S.E.M.) obtained by dose-inhibition analysis $\left(K_{\mathrm{i}}\right)$ and binding assay $\left(K_{0}\right)$ are listed. $K_{\mathrm{i}}$ values were obtained by fitting eqn. (1) to the dose-inhibition data (Figure 1) by non-linear least-squares regression. PP1/PP2A is the ratio of the $K_{\mathrm{i}}$ value for PP1 to that for PP2A. To estimate $K_{0}$ values, eqn. (20) was fitted to data from the binding assay (Figure 3) using eqn. (16) (general) or eqn. (19) (competitive) as the explicit form of $\phi$. In the fitting procedure using the general interaction model, the ratio of the dissociation constants $k$ [see eqn. (12)] was also estimated. The PP2A affinity of $\left[24-{ }^{3} \mathrm{H}\right] \mathrm{AA}$ was assumed to be the same as that of unlabelled $\mathrm{OA}$ (i.e. $K_{0}=K_{1}=32 \mathrm{pM}$ ). $n$ denotes the total number of experiments. See the text for further explanations.

\begin{tabular}{|c|c|c|c|c|c|c|c|c|c|c|}
\hline \multirow[b]{3}{*}{ Inhibitor } & \multicolumn{6}{|c|}{ Dose-inhibition analysis } & \multicolumn{4}{|c|}{ Binding assay (PP2A only) } \\
\hline & \multirow[b]{2}{*}{ Substrate } & \multicolumn{2}{|l|}{ PP1 } & \multicolumn{2}{|l|}{ PP2A } & \multirow[b]{2}{*}{ PP1/PP2A } & \multicolumn{2}{|l|}{ General } & \multicolumn{2}{|l|}{ Competitive } \\
\hline & & $K_{\mathrm{i}}(\mathrm{pM})$ & $n$ & $K_{\mathrm{i}}(\mathrm{pM})$ & $n$ & & $k$ & $K_{0}(\mathrm{pM})$ & $K_{0}(\mathrm{pM})$ & $n$ \\
\hline $\mathrm{OA}$ & $p N P P$ & $147000 \pm 34000$ & 48 & $32 \pm 2$ & 213 & 4600 & $2.6 \times 10^{8}$ & 32 (assumed) & 32 (assumed) & 86 \\
\hline \multirow[t]{2}{*}{ MCLR } & $\begin{array}{l}p N P P \\
P M I C\end{array}$ & $38 \pm 5$ & 58 & $8 \pm 1$ & 214 & 5 & $1.1 \times 10^{6}$ & $12 \pm 3$ & $12 \pm 3$ & 90 \\
\hline & $\begin{array}{l}\text { PMLC } \\
\text { Phosphorylase } a\end{array}$ & $50 \pm 22$ & $\begin{array}{l}48 \\
-\end{array}$ & $\begin{array}{r}13 \pm 8 \\
9 \pm 5\end{array}$ & $\begin{array}{l}72 \\
48\end{array}$ & $\begin{array}{r}4 \\
-\quad 4\end{array}$ & $\begin{array}{l}- \\
-\end{array}$ & - & - & - \\
\hline \multirow[t]{3}{*}{ Calyculin-A } & $p N P P$ & $1130 \pm 130$ & 40 & $126 \pm 30$ & 56 & 9 & $2.5 \times 10^{8}$ & $72 \pm 9$ & $72 \pm 12$ & 150 \\
\hline & PMLC & $980 \pm 260$ & 48 & $105 \pm 36$ & 48 & 7 & - & - & - & - \\
\hline & Phosphorylase $a$ & - & - & $113 \pm 42$ & 48 & - & - & - & - & - \\
\hline \multirow[t]{3}{*}{ Tautomycin } & $p N P P$ & $425 \pm 12$ & 60 & $33500 \pm 2200$ & 48 & 0.013 & $3.9 \times 10^{7}$ & $26500 \pm 3500$ & $26000 \pm 4400$ & 120 \\
\hline & PMLC & $480 \pm 60$ & 48 & $28600 \pm 4800$ & 48 & 0.017 & - & - & - & - \\
\hline & Phosphorylase $a$ & - & & $25800 \pm 6100$ & 48 & - & & & & \\
\hline
\end{tabular}

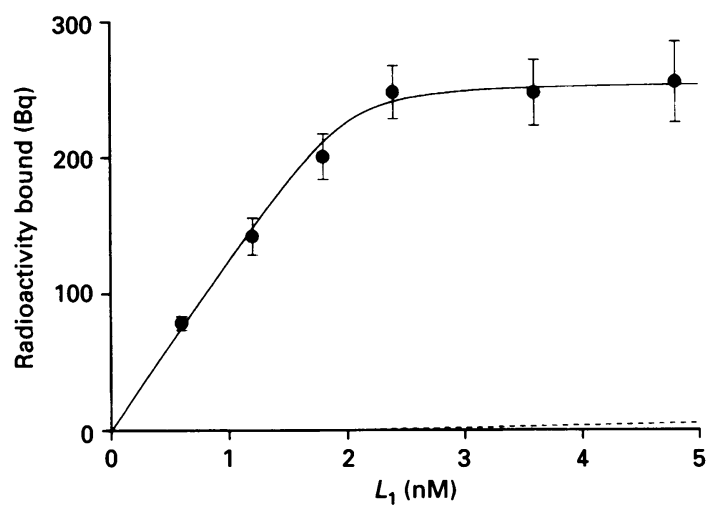

Figure 2 Binding of [24- $\left.{ }^{3} \mathrm{H}\right] \mathrm{OA}$ to PP2A

The specific binding of radioactivity to PP2A is shown as a function of the total concentration $L_{1}$ of $\left[24-{ }^{3} \mathrm{H}\right] \mathrm{OA}(0-4.8 \mathrm{nM})$. The molar concentration of PP2A was kept constant at $2.0 \mathrm{nM}$ on the basis of the $E$ value determined by dose-inhibition analysis with unlabelled $O A$. The solid curve is the least-squares fit of the theoretical function [eqn. (2)] to the data. The dashed line shows the estimated 'non-specific' binding of $\left[24^{3} \mathrm{H}\right] \mathrm{OA}$ to the filter [see eqn. (21)], which has been subtracted from the data. Each point represents the mean \pm S.E M. of six values. See the text for further explanations.

below). This phenomenon was not observed for the inhibition of PP1 by calyculin-A.

\section{Binding assay}

Figure 2 shows the relationship between the total concentration of $\left[24-{ }^{3} \mathrm{H}\right] \mathrm{OA}\left(L_{1}\right)$ and its binding to PP2A. The concentration of PP2A was fixed at $2.0 \mathrm{nM}$. Eqn. (20) with insertion of eqn. (2) as the explicit form of $\phi$ fitted the data well by least-squares regression, assuming that the $\mathrm{PP} 2 \mathrm{~A}$ affinity of $\left[24-{ }^{3} \mathrm{H}\right] \mathrm{OA}$ was the same as that of OA (i.e. $K_{1}=32 \mathrm{pM}$ ). The specific radioactivity, $N$, of our $\left[24-{ }^{3} \mathrm{H}\right] \mathrm{OA}$ obtained by this fitting procedure was estimated at $130 \mathrm{GBq} / \mathrm{mmol}$. The non-specific binding of radioactivity, $\epsilon$, which was also estimated by this procedure (Figure 2; dashed line), appeared to be relatively small in the concentration

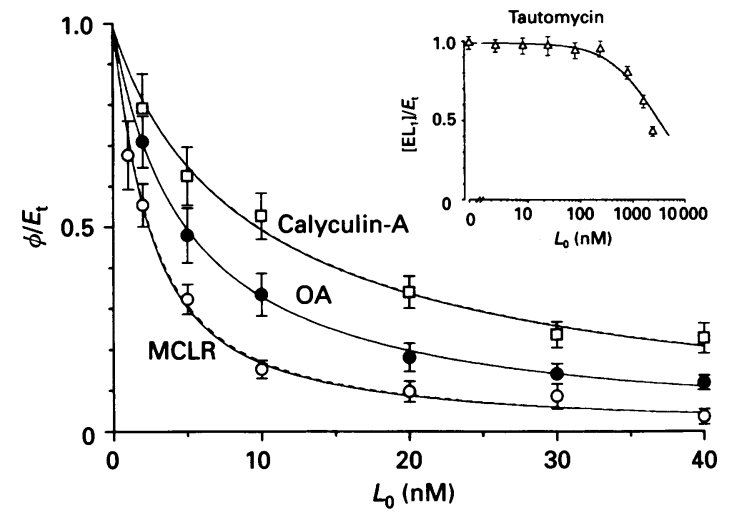

Figure 3 Binding of $\left[24-{ }^{3} \mathrm{H}\right] \mathrm{OH}$ to PP2A in competition with unlabelled
protein phosphatase inhibitors

Binding of $\left[24-{ }^{3} \mathrm{H}\right] \mathrm{OA}$ to PP2A was measured in the presence of various concentrations $\left(L_{0}\right)$ of unlabelled protein phosphatase inhibitors. Experiments were performed with constant concentrations of PP2A $(2.0 \mathrm{nM})$ and $\left[24{ }^{3} \mathrm{H}\right] \mathrm{OA}(5.0 \mathrm{nM})$. Under these conditions, the fraction of PP2A bound to $\left[24-{ }^{3} \mathrm{H}\right] \mathrm{OA}$ in the absence of unlabelled inhibitors is estimated by eqn. (1) at $99 \%$ of the total enzyme (cf. Figure 2). The binding was decreased by the unlabelled protein phosphatase inhibitors in a dose-dependent manner. Symbols for unlabelled inhibitors: $O$, MCLR;,$O A ; \square$, calyculin-A; $\triangle$, tautomycin. The ratio of the concentration of $\left[24{ }^{3} \mathrm{H}\right] \mathrm{OA}$ bound PP2A to the total enzyme concentration, $\phi / E_{\text {, }}$ is plotted against $L_{0}$ on a linear scale, except for the results obtained with tautomycin (inset) for which the scale of $L_{0}$ is logarithmic. Curves are least-squares fits of the theoretical functions derived for the general interaction model (- ) and competitive interaction model (----) respectively (see the Theory section). The non-specific binding of $\left[24^{-}{ }^{-} \mathrm{H}\right] \mathrm{OA}$ to filter, $\epsilon$, has been subtracted from the data. Symbols show means \pm S.E.M. The number of experiments are listed in Table 3, together with the values of $K_{0}$ and $k$ obtained by the fitting procedure.

range $(0-4.8 \mathrm{nM})$ of $\left[24-{ }^{3} \mathrm{H}\right] \mathrm{OA}$ used. Note that $\epsilon$ cannot be determined before the fitting because it is dependent on $\phi$ when $\mathrm{L}_{1}$ is a tight-binding ligand [see eqn. (21)]. Note also that the model function, eqn. (2), is mathematically equivalent to eqn. (4), on which the Scatchard analysis is based (see the Theory section).

As shown in Figure 3, the specific binding of $\left[24-{ }^{3} \mathrm{H}\right] \mathrm{OA}$ to 
PP2A was inhibited by addition of the unlabelled inhibitors in a dose-dependent manner. In these experiments, the total concentrations of PP2A and $\left[24-{ }^{3} \mathrm{H}\right] \mathrm{OA}$ in the reaction mixture were kept constant at $2.0 \mathrm{nM}$ and $5.0 \mathrm{nM}$ respectively. With this setting, the fraction of PP2A bound to $\left[24-{ }^{3} \mathrm{H}\right] \mathrm{OA}$ in the absence of unlabelled inhibitors is estimated by eqns. (2) and (3) at $99 \%$ of the total enzyme (see the Theory section). Thus the condition under which eqns. (16) and (19) may be used seems to be fulfilled. We tried first to fit the general interaction model (Scheme 1) to the data for MCLR, calyculin-A and tautomycin, using eqn. (16) as the explicit form of $\phi$ in eqn. (20). For the fitting, the dissociation constant for the PP2A interaction of $\left[24^{3} \mathrm{H}\right] \mathrm{OA}$ was again assumed to be the same as that of unlabelled OA (i.e. $K_{1}=32 \mathrm{pM}$ ). The regression curves obtained (Figure 3, solid lines) appear to fit the data points satisfactorily.

As shown in Table 3, the values of $k$ obtained by this fitting procedure were of the order of $10^{6}-10^{8}$. The very large $k$ values are indicative that the PP2A binding of $\left[24-{ }^{3} \mathrm{H}\right] \mathrm{OA}$ and that of the unlabelled inhibitors are mutually exclusive [see eqns. (11)-(13)]. Indeed, the competitive interaction model [Scheme 2; eqn. (19)] gave nearly the same regression curves (Figure 3; dashed lines) and $K_{0}$ values (Table 3 ) as did the general interaction model.

The concentration of $\left[24-{ }^{3} \mathrm{H}\right] \mathrm{OA}$ used in these binding assays had been adjusted beforehand by fitting eqn. (20) with the use of eqn. (18) as the explicit form of $\phi$ to several sets of $\left(L_{0}, \phi / E_{\mathrm{t}}\right)$ data obtained by trial assays with unlabelled OA as standard. [Eqn. (18) is suitable for this purpose because it is independent of $K_{0}$ and $K_{1}$.] Consequently, no regression procedure was required to fit the competitive interaction model to the data for OA shown in Figure 3, which were obtained by repeating similar experiments with the known concentration $(5 \mathrm{nM})$ of $\left[24-{ }^{3} \mathrm{H}\right] \mathrm{OA}$. What $k$ value is given by fitting the general interaction model when $\mathbf{L}_{0}$ and $L_{1}$ competes for the same site on a protein? Using eqn. (16) in place of eqn. (18) for fitting eqn. (20) to the same bindingassay data for OA, we obtained a value for $k$ of $2.6 \times 10^{8}$ (Table 3).

With MCLR, calyculin-A and tautomycin, there was no significant difference (at the 0.05 level) between the values of $K_{0}$ and those of $K_{1}$ for PP2A (Table 3). This also indicates that [24$\left.{ }^{3} \mathrm{H}\right] \mathrm{OA}$ and the unlabelled inhibitors bind to PP2A in a mutually exclusive manner. For calyculin-A, however, we obtained a $30-40 \%$ smaller value for $K_{0}$ then for $K_{i}$, although the difference $(P<0.1)$ was not judged as statistically significant at the 0.05 level because of the relatively large variation in $K_{1}$ data. In the dose-inhibition analysis with this inhibitor, the fractional activity of PP2A was more variable at medium inhibitor concentrations (see Figure 1). No such tendency appears to exist at any concentration of calyculin-A examined in the binding assay (Figure 3), where almost no free PP2A remains in the reaction mixture (see above). Therefore it seems reasonable to speculate that calyculin-A, a monophosphate ester, may be gradually hydrolysed by PP2A and loses its inhibitory activity towards the enzyme.

\section{DISCUSSION}

Several groups (MacKintosh et al., 1990; MacKintosh and Klumpp, 1990; Fujiki and Suganuma, 1993; Li and Casida, 1992) have reported that OA and some other protein phosphatase inhibitors prevent each other from binding to PP2A. However, little has been known until now about the kinetic properties of the interaction of the inhibitors on the enzyme molecule, mainly because of the technical difficulties related to the very high affinity of the inhibitors (see the Introduction). In the present paper we have introduced a procedure for examining to what extent the affinity of an enzyme, $\mathrm{E}$, for a labelled tight-binding ligand, $L_{1}$, is reduced when $\mathrm{E}$ binds to an unlabelled tightbinding ligand, $L_{0}$. Using this procedure we have analysed the dose-dependent reduction of PP2A (E) binding of $\left[24-{ }^{3} \mathrm{H}\right] \mathrm{OA}$ $\left(L_{1}\right)$ by addition of unlabelled protein phosphatase inhibitors $\left(\mathrm{L}_{0}\right)$ such as MCLR, calyculin-A and tautomycin (Figure 3 ). We have shown that very large values $\left(1.0 \pm 10^{6}-2.5 \times 10^{8}\right)$ must be assigned to $k$, the ratio of the dissociation constant for the $\mathrm{EL}_{0}-\mathrm{L}_{1}$ interaction to that for the $\mathrm{E}-\mathrm{L}_{1}$ interaction, for fitting the general interaction model (Scheme 1) to the binding-assay data (Table 3). This indicates that PP2A binding of $\left[24-{ }^{3} \mathrm{H}\right] \mathrm{OA}$ and unlabelled inhibitors are mutually exclusive [see eqn. (12)]. Indeed, almost the same regression curves (Figure 3) and $K_{0}$ values (Table 3 ) are obtained by the fitting of the competitive interaction model (Scheme 2) to the binding-assay data. The dissociation constants for the $\mathrm{E}-\mathrm{L}_{\mathbf{0}}$ interaction estimated by the binding assays, $K_{0}$, are in reasonable agreement with those obtained by dose-inhibition analysis, $K_{\mathrm{i}}$ (Table 3 ). The $k$ values for MCLR, calyculin-A and tautomycin approach the value $\left(2.6 \times 10^{8}\right)$ obtained by fitting the general interaction model to the binding-assay data for OA which probably interacts with $\left[24-{ }^{3} \mathrm{H}\right] \mathrm{OA}$ in a competitive manner.

These kinetic observations are most simply interpreted by assuming that the inhibitors compete for the same binding site. Alternatively, one could argue that binding might cause a conformational change in the enzyme molecule resulting in a decrease in affinity of the enzyme for a second inhibitor at a different binding site. In this case, however, the conformational change must be large enough to explain the very large reduction in the affinity. Eqn. (13) estimates that the decrease in the absolute values of the standard free-energy $\left(\delta \Delta G^{0 \prime}\right)$ for PP2A binding to $\left[24-{ }^{3} \mathrm{H}\right] \mathrm{OA}$ caused by the enzyme binding unlabelled ligands would be as large as $34-48 \mathrm{~kJ} \cdot \mathrm{mol}^{-1}$. These estimated $\delta \Delta G^{0 \prime}$ values amount to $56-80 \%$ of the absolute value of the standard free-energy change in PP2A binding of OA $\left[60 \mathrm{~kJ} \cdot \mathrm{mol}^{-1}\right.$ (Sasaki et al., 1994)]. Although it is logically difficult to exclude such an alternative interpretation completely, it seems reasonable to use the simple competitive-binding hypothesis as a starting point for further studies on the interaction of the protein phosphatase inhibitors on the PP2A molecule.

There are several lines of experimental evidence to show that interaction of inhibitors also occurs on the PP1 molecule (see MacKintoch et al., 1990). It should be noted that the principle of the method described in this paper may also be used to study the interaction of two tight-binding inhibitors on the PP1 molecule, if a labelled inhibitor with sufficiently high affinity for this enzyme is available. Unfortunately, $\left[24-{ }^{3} \mathrm{H}\right] \mathrm{OA}$ is not suitable for this purpose because the affinity of OA for PP1 is much lower than its affinity for PP2A (see Table 3). Eriksson et al. (1990) and Fujiki and Suganuma (1993) have examined binding of $\left[{ }^{3} \mathrm{H}\right]$ dihydroMCLR to tissue fractions. As the affinity of MCLR for PP1 is similar to that of OA for PP2A (Table 3), it will be interesting to examine binding of labelled MCLR to PP1 in the presence of unlabelled phosphatase inhibitors.

In the present experiments we have measured $K_{1}$ values for PP1 as well as those for PP2A. It is now well known that OA exhibits much higher affinity for PP2A than it does for PP1 [see e.g. Takai et al. (1992a)], whereas MCLR (MacKintosh et al., 1990) and calyculin-A (Ishihara et al., 1989) inhibit these enzymes with similar potency. These previous observations have been quantitatively confirmed by the present experiments (Table 3 ). The ratio of the $K_{1}$ for PP1 to that for PP2A (PP1/PP2A ratio) is about 5000 for OA, 5 for MCLR and 10 for calyculin-A. In contrast with the other three inhibitors examined, tautomycin 
shows markedly higher affinity for PP1 and for PP2A (Table 3). The PP1/PP2A ratio was $0.01-0.02$ for this inhibitor. This is considerably smaller than the value $(0.4)$ calculated from $K_{\mathrm{i}}$ values reported earlier (MacKintosh and Klumpp, 1990). At the present time, inhibitor proteins 1 and 2 (Nimmo and Cohen, 1978) are often used for selective inhibition of PP1. Compared with these peptides, which are not membrane-permeable, tautomycin, being hydrophobic, can enter the cell (Klumpp and Schultz, 1991). This property, together with its selectivity for PP1, will make tautomycin very useful for physiological experiments with intact cells.

Part of this work was performed while A. T. was working in the laboratory of Professor J. Caspar Rüegg (Heidelberg). We thank Mr. Josko Kuduz (Göttingen) and Ms. Monika Troschaka (Heidelberg) for their expert technical assistance. We are also grateful to Dr. Michio Murata (Tokyo) for useful discussions and to Dr. Lorraine M. Smith (Edinburgh and Nagoya) for reading the first and revised versions of the manuscript.

\section{REFERENCES}

Antoniw, J. F., Nimmo, H. G., Yeaman, S. J. and Cohen, P. (1977) Biochem. J. 162 423-433

Bialojan, C. and Takai, A. (1988) Biochem. J. 256, 283-290

Birkhoff, G. and MacLane, S. (1977) A Survey of Modern Algebra, Macmillan Publishing, New York

Carroll, R. J. and Ruppert, D. (1988) Transformation and Weighting in Regression, Chapman and Hall, New York

Cayla, X., Goris, J., Hermann, J., Hendrix, P., Ozon, R. and Merlevede, W. (1990) Biochemistry 29, 658-667

Cha, S. (1970) J. Biol. Chem. 245, 4814-4818

Cha, S. (1975) Biochem. Pharmacol. 24, 2177-2185

Cheng, X. C., Kihara, T., Kusakabe, H., Magae, J., Kobayashi, Y., Fang, R. P., Ni, Z. F.,

Shen, Y. C., Ko, K., Yamaguchi, I. and Isono, K. (1987) J. Antibiot. Tokyo 40, 907-909

Cheng, X. C., Ubukata, M. and Isono, K. (1990) J. Antibiot. Tokyo 43, 809-819

Cohen, P., Foulkes, J. G., Holmes, C. F. B., Nimmo, G. A. and Tonks, N. K. (1988) Methods Enzymol. 159, 427-437

Cohen, P. (1989) Annu. Rev. Biochem. 58, 453-508

Received 15 July 1994/7 November 1994; accepted 11 November 1994
Cummins, P. and Lambert, S. J. (1986) Circ. Res. 58, 846-858

Eriksson, J. E., Gronberg, L., Nygard, S., Slotte, J. P. and Meriluoto, J. A. (1990) Biochim. Biophys. Acta 1025, 60-66

Fujiki, H. and Suganuma, M. (1993) Adv. Cancer Res. 61, 143-194

Goris, J., Hermann, J., Hendrix, P., Ozon, R. and Merlevede, W. (1989) FEBS Lett. 245, 91-94

Henderson, P. J. F. (1972) Biochem. J. 127, 321-333

Ishihara, H., Martin, B. L., Brautigan, D. L., Karaki, H., Ozaki, H., Kato, Y., Fusetani, N., Watabe, S., Hashimoto, K., Uemura, D. and Hartshorne, D. J. (1989) Biochem. Biophys. Res. Commun. 159, 871-877

Klumpp, S. and Schultz, J. E. (1991) FEBS Lett. 288, 60-64

Li, Y. M. and Casida, J. E. (1992) Proc. Natl. Acad. Sci. U.S.A. 89, 11867-11870

Li, Y. M., MacKintosh, C. and Casida, J. E. (1993) Biochem. Pharmacol. 46, 1435-1443

Lowry, O. H., Rosebrough, N. J., Farr, A. L. and Randall, R. J. (1951) J. Biol. Chem. 193, 265-275

MacKintosh, C., Beattie, K. A., Klumpp, S., Cohen, P. and Codd, G. A. (1990) FEBS Lett. 264, 187-192

MacKintosh, C. and Klumpp, S. (1990) FEBS Lett. 277, 137-140

Matsushima, R., Yoshizawa, S., Watanabe, M. F., Harada, K., Furusawa, M., Carmichael, W. W. and Fujiki, H. (1990) Biochem. Biophys. Res. Commun. 171, 867-874

Ngai, P. K., Carruthers, C. A. and Walsh, M. P. (1984) Biochem. J. 218, 863-870

Nimmo, G. A. and Cohen, P. (1978) Eur. J. Biochem. 87, 353-365

Sasaki, K., Murata, M., Yasumoto, T., Mieskes, G. and Takai, A. (1994) Biochem. J. 298, 259-262

Scatchard, G. (1949) Ann. N.Y. Acad. Sci. 51, 660-672

Sen, A. and Srivastava, M. (1990) Regression Analysis: Theory, Methods, and Applications, Springer-Verlag, New York

Shenolikar, S. and Nairn, A. C. (1991) Adv. Second Messenger Phosphoprotein Res. 23, $1-121$

Snedecor, G. W. and Cochran, W. G. (1980) Statistical Methods, lowa State University Press, Ames, IA

Straus, 0. H. and Goldstein, A. (1943) J. Gen. Physiol. 26, 559-585

Takai, A., Bialojan, C., Troschka, M. and Rüegg, J. C. (1987) FEBS Lett. 217, 81-84

Takai, A. and Mieskes, G. (1991) Biochem. J. 275, 233-239

Takai, A., Murata, M., Torigoe, K., Isobe, M., Mieskes, G. and Yasumoto, T. (1992a) Biochem. J. 284, 539-544

Takai, A., Ohno, Y., Yasumoto, T. and Mieskes, G. (1992b) Biochem. J. 287, 101-106

Tung, H. Y., Resink, T. J., Hemmings, B. A., Shenolikar, S. and Cohen, P. (1984) Eur. J. Biochem. 138, 635-641

Wald, A. (1943) Trans. Am. Math. Soc. 54, 426-482 